\title{
Molecular dynamics study of the stability of aluminium coatings on iron
}

\author{
A. Ye. Galashev ${ }^{\dagger, 1,2}$, O. R. Rakhmanova ${ }^{1,2}$, V. A. $\operatorname{Kovrov}^{1}$, Yu. P. Zaikov ${ }^{1,2}$ \\ †alexander-galashev@yandex.ru
}

${ }^{1}$ Institute of High-Temperature Electrochemistry, UB RAS, 20 Akademicheskaya St., Yekaterinburg, 620990, Russia

${ }^{2}$ Ural Federal University n. a. the first President of Russia B. N. Yeltsin, 19 Mira St., Yekaterinburg, 620002, Russia

\begin{abstract}
Among the available protection systems for steel, the use of coatings is the most popular and economical method. One can protect the steel electrode from aggressive media with an aluminum coating. A thin $\mathrm{Al}$ film on an $\mathrm{Fe}$ substrate has been studied by the molecular dynamics method at a heating temperature from $300 \mathrm{~K}$ to $1500 \mathrm{~K}$. A significant horizontal displacement of individual $\mathrm{Al}$ atoms on the edges of the film is observed during the simulation. The film begins to "spread" slightly near the edges. This "spreading" creates the conditions for the beginning of diffusion of iron atoms into aluminum. Some $\mathrm{Al}$ atoms were found to penetrate the Fe matrix at a temperature of $873 \mathrm{~K}$. The total energy curve of the system shows both the melting transition in aluminum and phase transition from the body-centered cubic lattice to the face-centered cubic one at $1173 \mathrm{~K}$. The binding energy for the $\mathrm{Al}$ atom in the lattice of the $\mathrm{Fe}$ crystal is smaller than that for Fe atoms. The calculated diffusion coefficients for $\mathrm{Al}$ and $\mathrm{Fe}$ have a significantly slower growth with a temperature in the range of $673 \mathrm{~K} \leq T \leq 1500 \mathrm{~K}$. To describe the diffusion in a crystal using the molecular dynamics model, a temperature-dependent correction to the activation energy is calculated. The temperature dependence of the diffusion coefficient of aluminum atoms in an iron crystal can be represented as an Arrhenius expression with a temperature-dependent energy barrier for diffusion.
\end{abstract}

Keywords: aluminium film, diffusion coefficients, iron, molecular dynamics.

\section{Introduction}

The layer of aluminum coating on the reinforced steel has good resistance in intense corrosion environment. The mechanical properties of the specimen with coating in tensile testing remain stable. On the contrary, for the uncovered specimens, a decrease in strength properties, mass loss ratio, and ductility properties is observed [1]. Thus, the aluminum coating can serve as a good protection of steel against the influence of aggressive environment, containing, for example, water, oxygen, and halogens [2].

Both the properties of the solid material and the structure are determined by the solidifying process of the liquid alloy. For instance, the properties of light Al-based alloys change depending on temperature conditions [3]. Diffusion coefficients $D$ are important for understanding the solidifying processes, including crystal growth [4] and glass formation [5]. The diffusion coefficient of liquid $\mathrm{Al}$ is difficult to detect experimentally. The capillary tube method is usually used to measure directly diffusion coefficients in liquid alloys. This method requires isotopes, which are used as indicators. The effect of a convective flow existing along with the profile of diffusion during annealing, may lead to an overestimation of the value of $D$. Also, no radioactive isotopes are available for aluminum.

Aluminum, like the majority of other metals, is not resistant to corrosion for several reasons: humidity may preserve within the detail, bad welding or incorrect contact with other metals. Al-Mg and Al-Mn alloys exhibit good corrosion resistance. Extra pure $\mathrm{Al}(>99.9 \% \mathrm{Al})$ has the best anti-corrosion properties. The corrosion properties of alloys abruptly decrease when other metals (especially, $\mathrm{Cu}$ and $\mathrm{Fe}$ ) are added. The protective coatings of $\mathrm{Al}$ are applied in different ways to steel, cast iron, copper, titan, and brass. For instance, during the alitizing process (when steel is placed in liquid $\mathrm{Al}$ ), diffusion processes result in the formation of solid Al-Fe compounds on the surface of the steel. Near the liquidus temperature of $\mathrm{Al}-\mathrm{Fe}$ alloys (3.5-10 at.\% of $\mathrm{Fe}$ ), sedimentation of the $\mathrm{Al}_{3} \mathrm{Fe}$ crystals and their subsequent melting are observed [6]. A chemical compound of two or more metals (intermetallide) has a set ratio between the components.

Intermetallic compounds are of great interest due to their high-temperature strength, low density and high creep resistance [7]. The intermetallide layer at the interface grows during annealing [8]. However, intermetallides are not widely used because of their brittleness at room temperature [9]. The addition of a plastic metallic phase to the intermetallide provides a good combination of strength and impact resistance. The properties of such compounds are functions of the thickness of the intermetallic layer, structure and sequential location in a high-strength matrix. Reactive diffusion occurs between the alternatively placed layers of two different metals. The intermetallide layer at the interface grows during annealing. To optimize such properties as impact resistance and strength, it is important to understand the kinetics of the diffusion process. Nowadays there is a lack of data on the solid body diffusion in the Al-Fe system. 
Many processes occurring in metals and alloys (crystallization process, phase transformations, recrystallization, saturation processes of the surface by other components) are diffusive. Diffusion occurs very slowly in solids. This is due to the arrangement of atoms in the lattice sites around which they perform small thermal oscillations. The diffusion of iron in aluminum has been considered in several works [10-12]. Data on the diffusion of aluminum in iron are extremely rare and contradictory.

The present work is aimed to study the diffusion process at $\mathrm{Al}$ contact with $\mathrm{Fe}$, to analyze the temperature influence on the process, and to evaluate protective properties of aluminum for steel structures.

\section{Computer model}

$\mathrm{Al}$ and Fe cubic cells located vertically one above the other with a contacting face (100) served as the original simulation configuration. Also, the minimum distance between $\mathrm{Al}$ and $\mathrm{Fe}$ atoms was equal to the average distance between atoms in $\mathrm{Al}$ and Fe crystals. The top cell contained $864 \mathrm{Al}$ atoms packed into a face-centered cubic lattice. The bottom cell contained $1458 \mathrm{Fe}$ atoms of a body-centered cubic lattice. The initial size of the simulated cell was $2.5 \times 2.5 \times 4.7 \mathrm{~nm}$. The calculations were carried out in parallel for 7 temperatures in the range of $300-1500 \mathrm{~K}: 300,473,673$, $873,1000,1173,1500 \mathrm{~K}$. Aluminum is characterized by extra corrosion resistance and applicability, high thermal and electrical conductivities, but low mechanical properties. Aged aluminum has relatively poor fatigue properties compared to the other metals. This is due to the metastable structure under cyclic stresses. We aimed to determine the self-diffusion coefficient in an aluminum coating exposed to strong periodic stress during operation. In this case, artificial aging of the material may be performed by applying cyclic loads. The maximum cyclic loading $\sigma_{z z}^{0}$ in the axial direction achieved $300 \mathrm{MPa}$. The experimental axial elastic limit observed in [13] is $\sim 210 \mathrm{MPa}$, while the equivalent shear limit is only $\sim 140 \mathrm{MPa}$. In this work, the external axial load had a form $\sigma_{z z}=\sigma_{z z}^{0} \sin (\omega t)$ with a period of 1 ps. We used 10 load cycles of constant amplitude. Cyclic stress in the aluminum film was produced by applying the periodic force field $f_{z}$. The procedure for creating an external cyclic load stopped after 10 ps and the calculation continued. Here we do not take into consideration the formation and structure of cracks in detail. We aim to study the behavior of aged aluminum film on iron at low and high temperatures.

The Al crystal is found to crack. The upper part of the crystal almost separated from the thin crystalline $\mathrm{Al}$ film approximately after $5 \mathrm{mln}$ time steps at temperatures $T \leq 873 \mathrm{~K}$. Based on both this investigation and the data of [14], we can conclude that the brittle $\mathrm{Al}$ crystal cracked because $\mathrm{Al}$ atoms were pulled by $\mathrm{Fe}$ ones. This is also since there are differences in interatomic distances and asynchronous vibration of atoms in different subsystems. Then the separated upper part of the $\mathrm{Al}$ crystal was removed from each system. From $127(300 \mathrm{~K})$ to $144(873 \mathrm{~K})$ $\mathrm{Al}$ atoms remained on the $\mathrm{Fe}$ substrate. The duration of the new calculation (after removal of the upper part of the $\mathrm{Al}$ crystall) was $10 \mathrm{mln}$ time steps (1 ns) at each temperature.
No visible separation of the Al crystal was observed at a temperature of $1173 \mathrm{~K}$ after calculation under the cyclic load during 10 ps. Calculations at this temperature were performed for the whole system consisted of 2322 atoms $(864 \mathrm{Al}+1458 \mathrm{Fe})$. The normally used molecular dynamics models for $\mathrm{Al}$ of this size reproduce the properties of a bulk system (for example, elastic moduli) quite satisfactorily [15]. The molecular dynamics simulation time amounted 2 ns. In all cases, periodic boundary conditions (PBC) were executed only horizontally. Free boundary conditions were executed in other directions. The Finnis-Sinclair potential was used to describe the Al-Al, Fe-Fe and Al-Fe interactions [16]. This is justified from the viewpoint of the electron theory of the solid body. The Finnis-Sinclair potential can reproduce lattice constants, cohesive energies, elastic constant, vacancy formation energies, pressure-volume ratios, melting points and melting heats [17]. Moreover, for some fcc systems, for example, Al systems, lattice constants, cohesive energies and elastic constants of some alloys are reproduced by the used potential. They are comparable with the characteristics determined directly in $a b$ initio calculations.

The simplified model of the diffusion of atoms in any crystal is as follows: at low temperatures, an atom moves according to a common diffusion - vacancy mechanism. Collective diffusion is possible at high temperatures [18]. The differences of energies $E_{a}=E^{\star}-E_{0}$ between the initial state of the atom $E_{0}$ and its energy in the saddle point $E^{*}$ is defined as the activation energy of moving atoms. Usually, there is a basic state at zero temperature. In the crystal, the nodes of the ideal crystalline lattice are recognized as the basic state. The value $E_{a}$ is determined as the difference between the maximum and minimum potential energies of the whole system in the case of moving atoms from one interstitial site to another. The energy $E^{\star}$ has a stochastic nature. The diffusion coefficient of the atom in the crystal is calculated according to [19]

$$
D=D_{0} \exp \left(-\frac{E_{a}}{k T}\right),
$$

where $D_{0}$ is the constant independent of temperature $T, k$ is the Boltzmann constant.

In liquid or gas media, $D$ coefficient is calculated according to the changes in atoms locations in three-dimensional spatial coordinates by using Einstein's equation

$$
D=\lim _{t \rightarrow \infty} \frac{1}{6 t}\left\langle\frac{1}{N} \sum_{i=1}^{N}\left[\mathbf{r}_{i}(t)-\mathbf{r}_{i}\left(t_{0}\right)\right]^{2}\right\rangle,
$$

where $\mathbf{r}_{i}$ is the radius vector of $i$ atom, angle brackets denote averaging over the initial time $t_{0}$.

The activation energy $E_{a}$ may be defined according to

$$
E_{a}=\bar{E}+E_{f}-E_{0},
$$

where $\bar{E}$ is the average system energy at temperature $T, E_{f}$ is the energy fluctuations swing, $E_{0}$ is the energy of this system at $T=0 \mathrm{~K}$.

The diffusion barrier for carbon atoms in Fe, for example, in the temperature range $600-800 \mathrm{~K}$ is $0.77-0.90 \mathrm{eV}$ (molecular dynamics (MD) calculation). This is in agreement with the calculation using the density functional theory 
$(0.86 \mathrm{eV})$ [20]. At $400 \mathrm{~K} D=4.47 \times 10^{-17} \mathrm{~m}^{2} / \mathrm{s}$ according to MD calculations [21].

The modified LAMMPS code [22] for parallel computing in the applied MD method was used. The calculations were performed on a hybrid cluster calculator "Uran" at the Institute of Mathematics and Mechanics of the Ural Branch of RAS with a peak performance of $216 \mathrm{Tflop} / \mathrm{s}$ and $1864 \mathrm{CPU}$.

The steel electrode requires an $\mathrm{Al}$ coating for protection from the aggressive environment which causes Fe corrosion. Alitizing and electrolytic alitizing are general methods used to coat $\mathrm{Fe}$ with an $\mathrm{Al}$ film. Irrespective of the $\mathrm{Al}$ film formation technique, the pre-Fe surface should be cleaned off the oxide film. Annealing is performed to strengthen the Al film coating on the steel construction. This results in the metal atoms interdiffusion and the formation of a diffusion layer and an intermetallide layer. Fig. 1 demonstrates an Al film on a steel rod. The image was taken using a NEOFOT 32 microscope. The coating was formed by electrolysis at $T=1193 \mathrm{~K}$ with the following annealing [23]. Al (a bright spot in Fig. 1) in the form of separate dendrites penetrates the Fe lattice, and $\mathrm{Fe}$ penetrates inside the $\mathrm{Al}$ coating (dark blotches and darkening of the Al surface, facing the Fe substrate). Through this joint, the Al film is firmly fixed on the detail. The melting point of $\mathrm{Al}\left(T_{m}^{\mathrm{Al}}=933 \mathrm{~K}\right)$ is much lower than that of $\mathrm{Fe}\left(T_{m}^{\mathrm{Fe}}=1808 \mathrm{~K}\right)$.

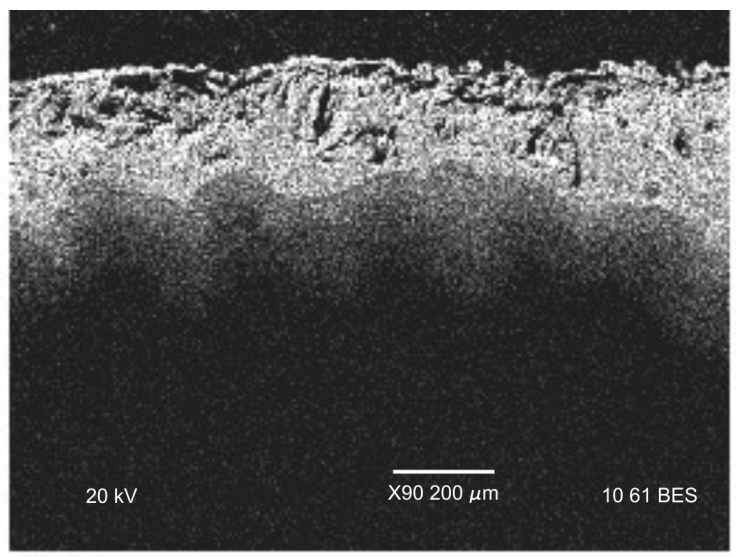

Al Ka1

Fig. 1. General view of the Al film (bright image) on the steel rod (dark part at the bottom) obtained by the electrolytic alitizing and annealing.

\section{Results}

Fig. 2 shows the configurations of the $\mathrm{Fe}-\mathrm{Al}$ system obtained after $10 \mathrm{mln}$ time steps at three temperatures: $300 \mathrm{~K}, 873 \mathrm{~K}$, and $1500 \mathrm{~K}$. At temperatures of $300 \mathrm{~K}$ and $873 \mathrm{~K}$, the behavior of the Al thin film on the Fe substrate was studied. At a temperature of $1500 \mathrm{~K}$, the Fe-Al system was modeled. Its initial configuration consisted of two massive parts of fcc Al and bcc Fe crystals. The atoms of both metals at a temperature of $300 \mathrm{~K}$ have low mobility, and no apparent view of mutual diffusion is observed. However, one can see significant horizontal displacements of individual $\mathrm{Al}$ atoms on the edges of the film. In other words, the film begins to "spread" slightly near the edges. This "spreading" creates the conditions for the beginning of $\mathrm{Fe}$ atoms diffusion into $\mathrm{Al}$. Mutual diffusion of $\mathrm{Al}$ and $\mathrm{Fe}$ atoms is observed at $873 \mathrm{~K}$.

In this case, the view of the two-component system has changed significantly. The system expanded and "sagged". The number of $\mathrm{Al}$ atoms on the (001) surface of bcc Fe greatly decreased, since their noticeable part penetrated inside the Fe crystal. Several Fe atoms appeared on the upper surface of the system. In a two-component system, as a result of structural rearrangement, a new crystalline order is formed. The presence of crystalline ordering of the atoms on the righthand side of the system at $T=873 \mathrm{~K}$ can be seen in Fig. 2 . The resulting bcc Fe crystal has substitution defects in the form of individual $\mathrm{Al}$ atoms in the lattice sites.

The Fe crystal expanded at $1500 \mathrm{~K}$. Intensive mutual diffusion of $\mathrm{Al}$ and $\mathrm{Fe}$ atoms was observed. The entire volume of the expanded Fe crystal was filled with $\mathrm{Al}$ atoms. They not only replaced Fe atoms in the crystal, but also occupied the interstices (Fig. 2). It is very difficult to detect visually the structural phase transition in Fe occurring at a temperature of $1173 \mathrm{~K}$. Nevertheless, the dependence of the total energy on the temperature at $T=1173 \mathrm{~K}$ undergoes a kink. Such a transition occurs only in the interior of the Fe subsystem and is confined to a relatively small region. The rapid diffusion of $\mathrm{Al}$ atoms into $\mathrm{Fe}$ prevents the complete rearrangement of the Fe subsystem in the fcc structure. In this case, the density of the bcc packing is lower than that of the fcc one. So, $\mathrm{Al}$ atoms pass faster through the bcc crystal, replacing the Fe atoms in
$T=300 \mathrm{~K}$

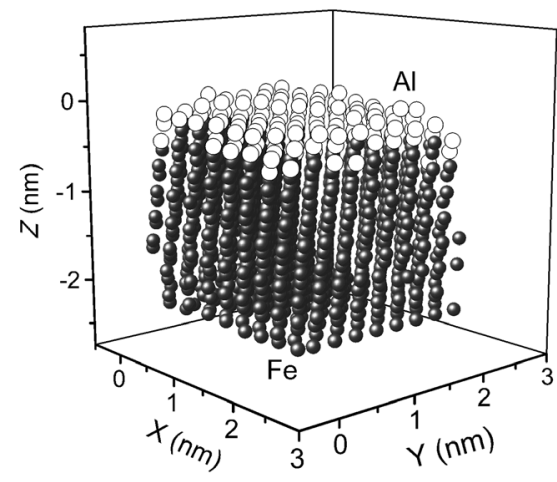

$T=873 \mathrm{~K}$

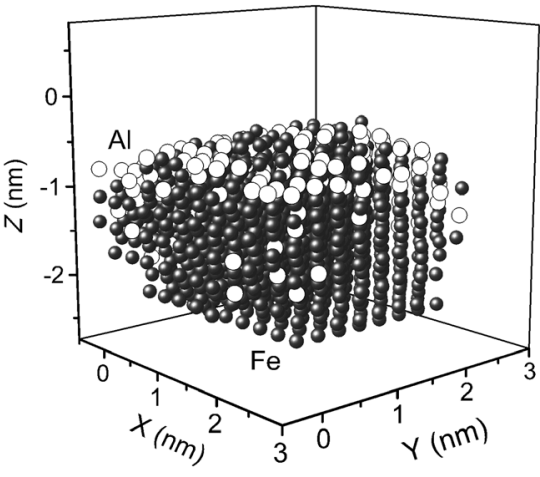

$T=1500 \mathrm{~K}$

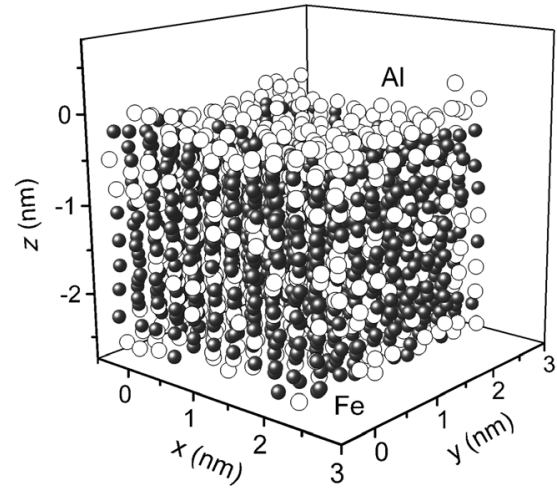

Fig. 2. "Al film on Fe substrate" system configurations obtained by the time moment of $1 \mathrm{~ns}$ at different temperatures. 
this package. The density $\left(5.903 \mathrm{~g} / \mathrm{cm}^{3}\right)$ of the $\mathrm{Fe}-\mathrm{Al}$ system at $T=1500 \mathrm{~K}(t=1 \mathrm{~ns})$ becomes lower than that of the initial bcc crystal of iron $\left(7.874 \mathrm{~g} / \mathrm{cm}^{3}\right)$ at $T=300 \mathrm{~K}$. Al atoms can be found in different parts of each plane, but their distribution is not homogeneous.

The temperature dependence $U_{\text {tot }}(T)$ of the total energy of $\mathrm{Al}$ and $\mathrm{Fe}$ atoms is shown in Fig. 3. A monotonous increase in the energy $U_{\text {tot }}$ is seen until $T=873 \mathrm{~K}$. The $U_{\text {tot }}$ energy increases sharply in the range of $873 \mathrm{~K} \leq T \leq 1500 \mathrm{~K}$. This behavior of the $U_{\text {tot }}(T)$ function indicates the presence of structural rearrangement. This is associated with the melting of the aluminum subsystem $(T=873 \mathrm{~K})$ and the replacement of $\mathrm{Fe}$ atoms by $\mathrm{Al}$ atoms in the system at temperatures of $T \geq 873 \mathrm{~K}$.

The dashed line in Fig. 3 denotes the asymptotic extension of the $U_{t o t}(T)$ function to the low-temperature region. The point of contact between the function extension and the $Y$-axis provides the $U_{0}$ value. It corresponds to the value of total energy $U_{\text {tot }}$ at absolute zero. The difference $U_{\text {tot }}-U_{0}$ defines the activation energy of defect formation in the Fe matrix and Al crystalline film. The dash-dot line in Fig. 3 shows the approximation dependence of $U_{\text {tot }}(T)$ obtained in the temperature interval $0 \leq T \leq 1500 \mathrm{~K}$. The approximation equation for $U_{\text {tot }}(T)$ function (exponential growth) is

$$
U=U_{1} \exp \left(\frac{T}{T_{1}}\right)+U_{0},
$$

where $U_{0}=-3.98701 \mathrm{eV}, U_{1}=0.06866 \mathrm{eV}, T_{1}=467.0 \mathrm{~K}$. The inset shows the energy change in time $U_{\text {tot }}$ at $T=300 \mathrm{~K}$. It is seen that the equilibrium of the system is achieved after 0.4 ns of simulation. We assume that the value of $E_{f}$ in expression (3) is constant. The $E_{f}$ quantity can be considered as a diffusion barrier, which is also related to the displacement of atoms to the saddle point. Such fluctuations in the location of atoms can be realized in the MD experiment only at high temperatures $(T \geq 673 \mathrm{~K})$.

Assuming $T=0$ in Eq. (4), we find the energy value $\bar{U}_{\text {tot }}^{0}=3.91835 \mathrm{eV}$, which is used to determine the temperaturedependent part of the energy barrier $\Delta E_{a}=\bar{U}_{t o t}(T)-\bar{U}_{\text {tot }}^{0}$ for diffusion. Here $\bar{U}_{t o t}(T)$ is the total energy of the system at temperature $T$. The $\Delta E_{a}$ value can reach a value that represents an appreciable part of the $E_{a}$ entire energy barrier. The $\Delta E_{a}$ correction to the activation energy amplifies with increasing temperature. So, the $\Delta E_{a}$ value is $0.06 \mathrm{eV}$ at $T=300 \mathrm{~K}$ and $\Delta E_{a}=1.63 \mathrm{eV}$ at $T=1500 \mathrm{~K}$. The $\Delta E_{a}$ correction should be subtracted from the energy barrier calculated by the MD method. In other words, the calculated barrier for diffusion should be reduced. The need for such a correction is since with increasing temperature model crystal structures continue to remain largely perfect structures that have neither point defects nor extended ones. And the higher the temperature, the greater the number of defects present in the crystal.

Fig. 4 shows the $\log D(1000 / T)$ dependence calculated in the MD simulation and the corrected dependence using the Arrhenius formula. Here we also give a comparison with the corresponding experimental dependence for the diffusion of iron in aluminum [10]. Analogous dependences showing both the temperature behavior of the diffusion coefficient in liquid aluminum [24] and $\alpha$ - and $\gamma$-phases of the solid Fe [25] are also presented in Fig. 4. The Curie temperature for $\mathrm{Fe}$ is $1043 \mathrm{~K}$. The experimental temperature of the bcc-fcc structural transition is $1183 \mathrm{~K}$. The calculated coefficients $D$ for $\mathrm{Al}$ and $\mathrm{Fe}$ have a significantly slower growth with a temperature in the range of $673 \mathrm{~K} \leq T \leq 1500 \mathrm{~K}$. The obtained growth rate of the $D$ value with temperature corresponds to a similar characteristic for molten aluminum obtained experimentally. The activation energy for diffusion depends on the temperature. This leads to nonlinearity in the $\log D(1000 / T)$ dependence obtained in the MD calculations. A particularly strong change in the behavior of this dependence occurs at a temperature of $1173 \mathrm{~K}$, i.e. in the field of the bcc-fcc structural transition. In this case, owing to the structural rearrangements occurring during the MD simulation, an increase in the diffusion coefficient of $\mathrm{Al}$ atoms in $\mathrm{Fe}$ is observed. At the same time, the data obtained in the MD calculations indicate a less rapid decrease in $D$ value with decreasing temperature. This can be seen from the $\log D(1000 / T)$ dependence calculated for the diffusion of $\mathrm{Fe}$ atoms in aluminum [10]. The temperature dependence of the $E_{a} / k T$ value calculated by us is shown in the inset to Fig. 4.

The activation energy for diffusion is determined as $E_{a}=E_{a}^{\prime}-\Delta E_{a}$, where $E_{a}^{\prime}=1.5543 \mathrm{eV}$ is the activation energy calculated using the Arrhenius approximation of the $D(T)$ dependence obtained in the MD simulation. The $D_{0}$ coefficient in the expression represented by the Arrhenius formula is $6.9831 \cdot 10^{-7} \mathrm{~m}^{2} / \mathrm{s}$. Thus, the obtained temperature dependence of the diffusion coefficient of $\mathrm{Al}$ atoms in the $\mathrm{Fe}$ crystal has the form

$$
D=D_{0} \exp \left[-\left(1.5543(\mathrm{eV})-\Delta E_{a}(T)\right) /(k T)\right],
$$

where $\Delta E_{a}$ value within the temperature range is expressed as

$$
\Delta E_{a}(\mathrm{eV})=0.0686(\mathrm{eV}) \cdot \exp \left(\frac{T}{467(\mathrm{~K})}\right)-0.0685(\mathrm{eV}),
$$

The $\Delta E_{a}(T)$ dependence calculated by the MD simulation and its approximation by the Eq. (6) are given in Fig. 5. As can be seen from Fig. 5, Eq. (6) serves as a good approximation for the function $\Delta E_{a}(T)$.

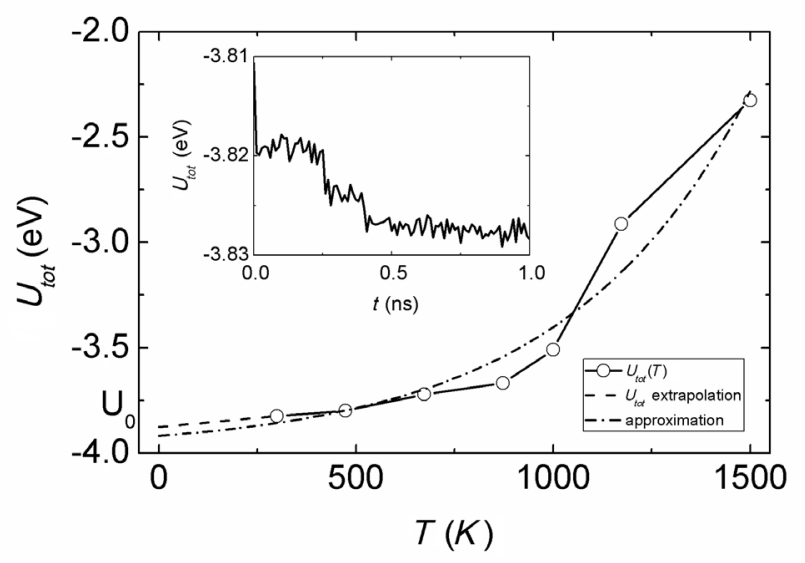

Fig. 3. Temperature dependence of the total energy of the "Al film on Fe substrate" system. The dash-dotted line denotes the approximation of the $U_{\text {tot }}(T)$ function by means of the Eq. (4). The insertion presents the time change in the system potential energy at $T=300 \mathrm{~K}$. 


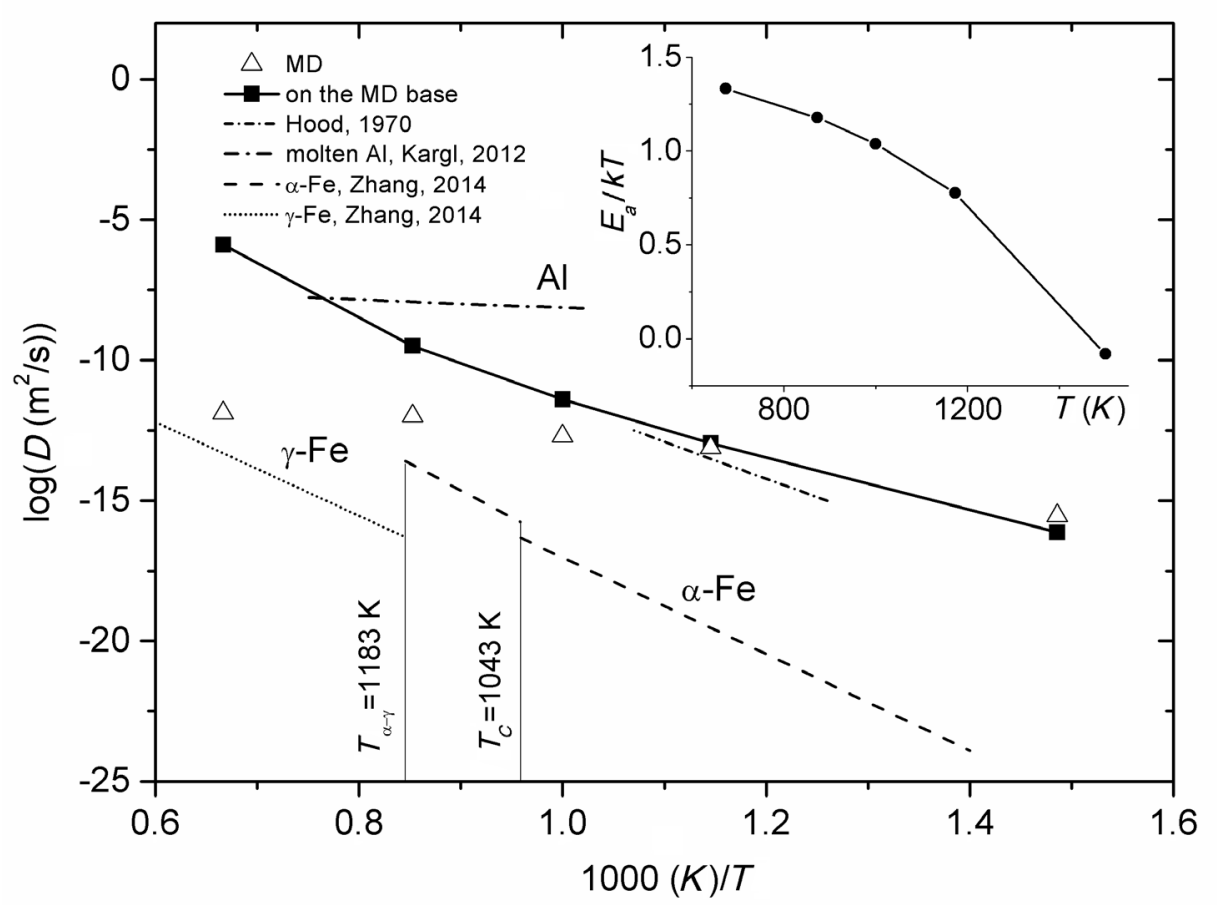

Fig. 4. The temperature dependence of $\mathrm{Al}$ diffusion in Fe obtained in the MD simulation and with using the MD data and the Arrhenius formula as well as the $D(T)$ dependences for molten aluminum and $\alpha$ - and $\gamma$-iron. The experimental dependence of the diffusion coefficient of $\mathrm{Fe}$ in $\mathrm{Al}$ is also given for comparison. The relative activation energy for the diffusion of $\mathrm{Al}$ into $\mathrm{Fe}$ is given in the inset.

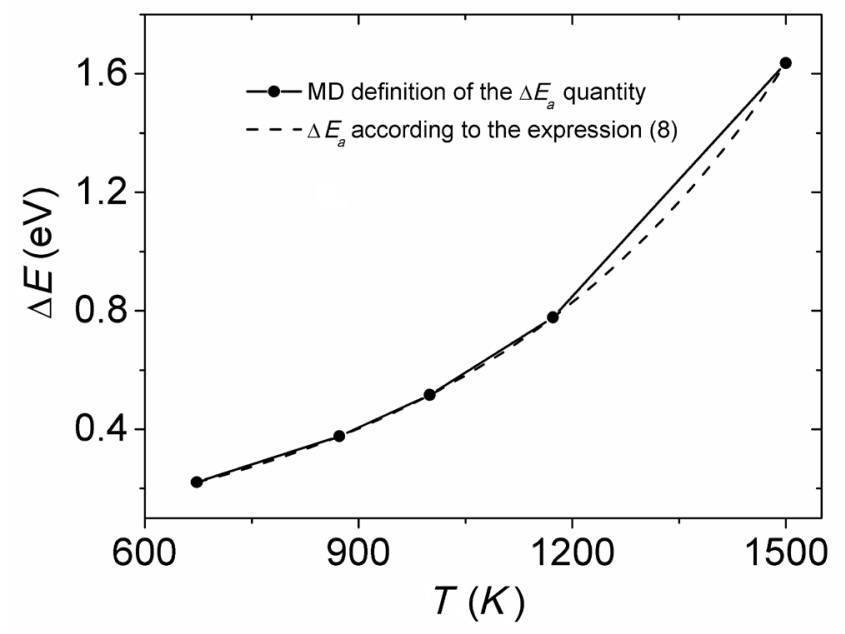

Fig. 5. The $\Delta E_{a}(T)$ function calculated by the MD method and its approximation by means of the Eq. (6).

\section{Conclusion}

The formation of an aluminum film on a steel product acts as a diffusion barrier for oxygen and the impact of aggressive environment. The main mechanism of diffusion of $\mathrm{Al}$ in Fe is diffusion over vacancies with the formation of a solid substitutional solution. The binding energy for the $\mathrm{Al}$ atom in the lattice of the Fe crystal is smaller than that for the basic atoms $(\mathrm{Fe})$. So, hetero-diffusion predominates self-diffusion, i. e. $D_{\mathrm{Al}}>D_{\mathrm{Fe}}$. The formation of vacancies near foreign atoms is facilitated. As a result, diffusion occurs in the form of the formation and vanishing of the complex "foreign atom vacancy". An increase in the $D$ coefficient at diffusion of $\mathrm{Al}$ in $\mathrm{Fe}$ with temperature can be represented by the formula $\left.D=6.9831 \cdot 10^{-7} \cdot \exp \left[-\left(1.5543(\mathrm{eV})-\Delta E_{a}(T)\right) / k T\right)\right]$.
Here, the temperature-dependent correction to the energy barrier for diffusion is given by the expression $\Delta E(\mathrm{eV})=0.0686(\mathrm{eV}) \cdot \exp (T / 467(\mathrm{~K}))-0.0685(\mathrm{eV})$, $673 \mathrm{~K} \leq T \leq 1500 \mathrm{~K}$. According to the expression for $D(T)$ function, $D(T)$ grows insignificantly in comparison with the behavior of the experimental $D(T)$ dependence for $\alpha$-iron in the temperature region of $673 \mathrm{~K} \leq T \leq 1173 \mathrm{~K}$. But it reproduces the growth rate of $D(T)$ function for $\gamma$-iron at $T>1173 \mathrm{~K}$.

\section{References}

1. G. Diamantogiannis, Ch.Alk. Apostolopoulos, P. G. Nikolakopoulos. J. Mater. Civ. Eng. 27, 1094 (2015). Crossref

2. A.E. Galashev, O.R. Rakhmanova, O.A. Novruzova. High Temp. 49, 528 (2011). Crossref

3. N. Jakse, A. Pasture. Scientific Reports. 3, 3135 (2013). Crossref

4. G. Kasperovich, A. Meyer, L. Ratke. Int. Found. Res. 62, 8 (2010).

5. A. Inoue. Acta Mater. 48, 279 (2000). Crossref

6. A.L. Beltukov, S. G. Menshikova, V.I. Ladyanov. High Temp. 53, 491 (2015). Crossref

7. J.N. Kim, C.S. Lee, Y.S. Jin. Met. \& Mater. Intern. 24, 1090 (2018). Crossref

8. S. Joung, W. Nam. Met. \& Mater. Intern. 25, 34 (2019). Crossref

9. N.S. Stoloff, C. T. Liu, S. C. Deevi. Intermetallics. 8, 1313 (2000). Crossref

10. G. M. Hood. Phil. Mag. 21, 305 (1970). Crossref

11. V. Jindal, V.C. Srivastava, A. Das, R. N. Ghosh. Mater. Lett. 60, 1758 (2006). $\underline{\text { Crossref }}$ 
12. B. Gao, S. Nalano, K. Kakimoto. Cryst. Growth Des. 12, 522 (2011). Crossref

13. A. May, L. Taleb, M. A. Belouchrani. 13th Intern. Conf. on Fracture. Beijing, China (2013) p. 1.

14. A.E. Galashev. Tech. Phys. 59, 467 (2014). $\underline{\text { Crossref }}$

15. R. Komanduri, N. Chandrasekaran, L.M. Raff. Int. J. Mech. Sci. 43, 2237 (2001). Crossref

16. M.W. Finnis, J.E. Sinclair. Phil. Mag. A. 50, 45 (1984). Crossref

17. A.E. Galashev, L.A. Elshina. R. V. Muradymov. Rus. J. Phys. Chem. A. 90, 2444 (2016). Crossref

18. V.V. Alekseenko. Phys. Solid State. 50, 1848 (2008). Crossref
19. I. I. Novoselov, A. Yu. Kuksin, A. V. Yanilkin. Phys. Solid State. 56, 1025 (2014). Crossref

20. T. T. Lau, C. J. Först, X. Lin, J. D. Gale, S. Yip, K. J. van Vliet. Phys. Rev. Lett. 98, 2155011 (2007). Crossref

21. A. Ishii, S. Ogata, H. Kimizuka. Phys. Rev. B. 85, 0643031 (2012). Crossref

22. S. J. Plimpton. Comp. Phys. 117, 1 (1995). Crossref

23. Yu. P.Zaikov, V. A. Kovrov, I. V. Brodova, Yu. N. Shtefanyuk, V. K. Pingin, D. I. Vinogradov. Adv. Mater. Res. 1088, 250 (2015). Crossref

24. F. Kargl, H. Weis, T. Unruh, A. Meyer. J. Phys.: Conference Series. 340, 012077 (2012). $\underline{\text { Crossref }}$

25. B. Zhang. AIP Advances. 4, 017128 (2014). $\underline{\text { Crossref }}$ 